\title{
Electrooptic Modulators with Controlled Frequency Responses by Using Nonperiodically Polarization-Reversed Structure
}

\author{
Ha Viet Pham, Hiroshi Murata, and Yasuyuki Okamura \\ Graduate School of Engineering Science, Osaka University, Machikaneyama, Toyonaka, Osaka 560-8531, Japan \\ Correspondence should be addressed to Ha Viet Pham, havietpham@ec.ee.es.osaka-u.ac.jp
}

Received 20 June 2008; Accepted 2 September 2008

Recommended by Chang-qing Xu

We discuss a new method to design traveling-wave electrooptic modulators with controlled frequency responses using nonperiodically polarization-reversed structure. Using our method, the frequency responses of both magnitude and phase of modulation index are controllable. Several electrooptic modulators for advanced modulation formats such as duobinary modulation and wideband single-sideband modulation are proposed.

Copyright () 2008 Ha Viet Pham et al. This is an open access article distributed under the Creative Commons Attribution License, which permits unrestricted use, distribution, and reproduction in any medium, provided the original work is properly cited.

\section{Introduction}

High-speed traveling-wave electrooptic modulators are very important devices because of their applications in many optoelectronic fields, such as telecommunication, optical signal processing, and optical measurement. With the increasing demand for broadband communication, many studies on the high-speed electrooptic modulators have been carried out for optical fiber communication system [1]. In addition, the precise control of the frequency responses of high-speed electrooptic modulators has been required for the new generation optical fiber communication systems [24]. In a standard traveling-wave electrooptic modulator, the frequency response of the modulation index is restricted by two effects: the velocity mismatching between the lightwave and modulation microwave and the loss of modulation microwave in the traveling-wave electrodes.

The polarization reversal technology of ferroelectric optical crystals, such as $\mathrm{LiNbO}_{3}$ and $\mathrm{LiTaO}_{3}$, is attractive for realizing high-performance electrooptic modulators. We have developed traveling-wave electrooptic modulators utilizing the periodically polarization-reversed structure for quasivelocity-matching (QVM) between the lightwave propagating in the optical waveguide and the modulation microwave traveling along the electrodes. This technique is attractive because it compensates for the velocity mismatch in the high frequency ranges without the requirement of specific and fine structures of the optical waveguide and the electrodes for velocity matching. Several advanced electrooptic modulators using periodically polarization-reversed structure have been proposed and demonstrated, such as a QVM electrooptic phase modulator [5], an electrooptic single-sideband (SSB) modulator [6], and an optical frequency shifter [7]. The bandwidth of the modulation frequency response in these electrooptic modulators with the simple periodically polarization-reversed structure is inversely proportional to its electrode length and becomes relatively narrow for a longelectrode device.

We have proposed a design to realize the travelingwave electrooptic modulators utilizing nonperiodically polarization-reversed structures [8]. Using the proposed design, it is possible to obtain electrooptic modulators with the arbitrary frequency responses of the magnitude of the modulation index over a specified frequency range. The flat broadband electrooptic modulator with the compensation for the degradation by both velocity mismatch and the microwave loss is obtained. However, the control of not only the magnitude but also the phase of the modulation index is very important in order to design the electrooptic modulators for advanced modulation formats.

In this paper, we extend our design method to control frequency responses of both the magnitude and the phase of modulation index in electrooptic modulators with nonperiodically polarization-reversed structures utilizing 


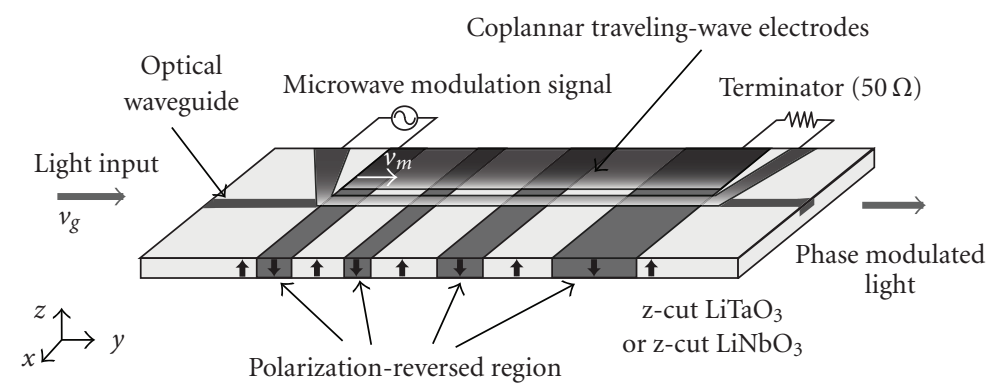

FiguRE 1: Basic structure of traveling-wave electrooptic phase modulator with nonperiodically polarization-reversed structure.

new flatness parameters. This new approach is applicable to the electrooptic modulators for advanced modulation formats, such as duobinary modulation or broadband singlesideband modulation. This paper is organized as follows. In Section 2, the basic structure of the electrooptic phase modulator using the nonperiodic polarization reversal and the frequency responses of modulation index are presented. In Section 3, the calculation method to control the frequency responses of both the magnitude and the phase of the modulation index is described. Several calculation results are shown in Section 4. In Section 5, the designs of the duobinary modulator and broadband single-sideband modulator with nonperiodically polarization-reversed structure are discussed.

\section{Traveling-Wave Electrooptic Modulators with Polarization-Reversed Structure}

A schematic of the basic structure of the traveling-wave electrooptic modulator is shown in Figure 1. It consists of a single-mode channel waveguide and simple coplanar traveling-wave electrodes fabricated on a ferroelectric substrate. A Mach-Zehnder waveguide for intensity modulation is also applicable. The velocity mismatch between the group velocity of the lightwave propagating in the optical waveguide and the phase velocity of a modulation microwave traveling along the electrodes is compensated for by adopting the periodic polarization reversal scheme [9]. The domain length $L$ of a standard QVM with the periodic polarization reversal scheme is determined as follows:

$$
L=\frac{1}{2 f_{m}\left(1 / v_{m}-1 / v_{g}\right)},
$$

where $f_{m}$ is the peak modulation frequency, $v_{m}$ is the phase velocity of the modulation microwave traveling along the electrodes, and $v_{g}$ is the group velocity of the lightwave propagating in the waveguide. The parameter $v_{m}$ can be calculated from the effective dielectric constant of the coplanar traveling-wave electrodes taking account of its structure. The parameter $v_{g}$ can be derived from both the material dispersion of the refractive index of the ferroelectric material substrate and the mode dispersion of the optical waveguide.
The nonperiodic polarization reversal scheme is used to control the frequency response of the electrooptic modulation. Figure 2 shows the structure of the nonperiodic polarization reversal. The total active length for modulation is $L_{t}$. It is divided into $M$ sections of successive polarizationreversed and nonreversed region. Each domain length is $L_{i}(i=1,2, \ldots, M)$.

The modulation index $\Delta \phi(f)$, which is a function of the modulation microwave frequency $f$, is calculated using the following equation:

$$
\begin{aligned}
\Delta \phi(f)= & -\int_{0}^{y_{1}} k \Delta n d y+\int_{y_{1}}^{y_{2}} k \Delta n d y \\
& -\cdots+(-1)^{M} \int_{y_{M-1}}^{y_{M}} k \Delta n d y,
\end{aligned}
$$

where $k$ is the wave vector of the lightwave in vacuum and $\Delta n$ is the refractive index change by the Pockels effect. When the light polarization is along the $z$-axis and the modulation electrical field is also along the $z$-axis, the refractive index change induced by the electrical field of the microwave at the position $y\left(0 \leq y \leq L_{t}\right)$ is derived as follows, taking account of the velocity mismatch between the lightwave and the modulation wave,

$$
\begin{aligned}
\Delta n(y)= & \frac{1}{2} n_{e}^{3} r_{33} \frac{V}{d} \Gamma \exp (-\alpha y) \\
& \times \exp \left[j 2 \pi f\left\{t_{0}-\left(\frac{1}{v_{m}}-\frac{1}{v_{g}}\right) y\right\}\right],
\end{aligned}
$$

where $n_{e}$ is the extraordinary ray refractive index of the substrate, $r_{33}$ is the Pockels coefficient of the substrate. $V$ is the voltage applied to the electrodes, $d$ is the gap between the electrodes, $\Gamma$ is the overlap factor between the optical field and the electrical field, $\alpha$ is the attenuation coefficient of the electrical field of the modulation microwave, and $t_{0}$ is the time when the lightwave arrived at the position $y=0$.

\section{Control of Frequency Response}

When tuning the length of each domain, the frequency responses of the modulation index magnitude $|\Delta \phi(f)|$ and phase $\arg [\Delta \phi(f)]$ change. In order to design a nonperiodic polarization reversal structure for an arbitrary frequency 


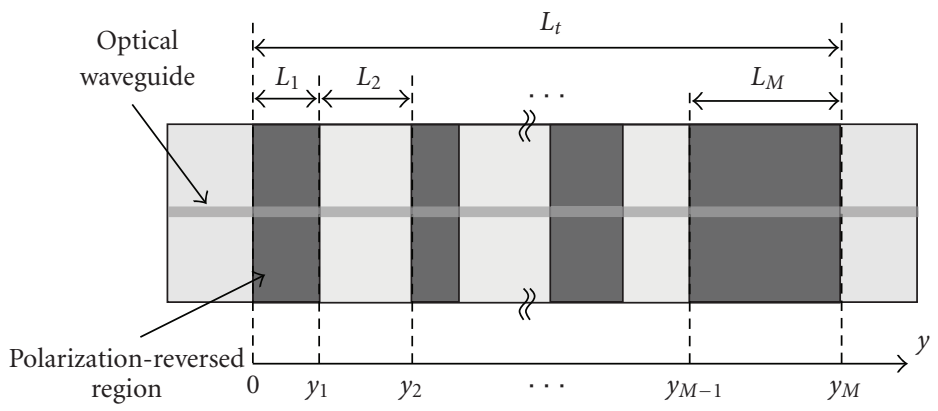

FIGURE 2: Nonperiodically polarization-reversed structure.

response of the modulation index magnitude, we have proposed the modified flatness parameter MF [8], which expresses the difference between the magnitude of the modulation index $|\Delta \phi(f)|$ and the target function $\operatorname{TF}(f)$ in the defined frequency range from $f_{1}$ to $f_{2}$

$$
\mathrm{MF}=\frac{\left(f_{2}-f_{1}\right) \int_{f_{1}}^{f_{2}}|\Delta \phi(f) / \mathrm{TF}(f)|^{2} d f}{\left[\int_{f_{1}}^{f_{2}}|\Delta \phi(f) / \mathrm{TF}(f)| d f\right]^{2}}-1
$$

By tuning the length of each domain, the value of the modified flatness parameter changes. If the modified flatness parameter becomes zero, the magnitude of the modulation index and the target function completely coincide in the frequency range.

We extended this method for dealing with both the modulation index magnitude and the phase. We define two flatness parameters, $\mathrm{MF}_{M}$ and $\mathrm{MF}_{P}$, for the magnitude and the phase of the modulation index by using two target functions: $\operatorname{TF}_{M}(f)$ for the magnitude and $\operatorname{TF}_{P}(f)$ for the phase in the defined frequency range from $f_{1}$ to $f_{2}$, as follows:

$$
\begin{gathered}
\mathrm{MF}_{M}=\frac{\left(f_{2}-f_{1}\right) \int_{f_{1}}^{f_{2}}\left(|\Delta \phi(f)|^{2} /\left|\mathrm{TF}_{M}(f)\right|^{2}\right) d f}{\left[\int_{f_{1}}^{f_{2}}\left(|\Delta \phi(f)| /\left|\mathrm{TF}_{M}(f)\right|\right) d f\right]^{2}}-1, \\
\mathrm{MF}_{P}=\frac{\left(f_{2}-f_{1}\right) \int_{f_{1}}^{f_{2}}\left(|\arg [\Delta \phi(f)]|^{2} /\left|\mathrm{TF}_{P}(f)\right|^{2}\right) d f}{\left\{\int_{f_{1}}^{f_{2}}\left(|\arg [\Delta \phi(f)]| /\left|\mathrm{TF}_{P}(f)\right|\right) d f\right\}^{2}}-1,
\end{gathered}
$$

where $\mathrm{MF}_{M}$ is the difference between the magnitude of the modulation index $|\Delta \phi(f)|$ and $\mathrm{TF}_{M}(f)$, and $\mathrm{MF}_{P}$ is the difference between the phase of the modulation index $\arg [\Delta \phi(f)]$ and $\mathrm{TF}_{P}(f)$.

If the modified flatness parameter $\mathrm{MF}_{M}$ becomes zero, the magnitude of the modulation index $|\Delta \phi(f)|$ and the target function $\mathrm{TF}_{M}(f)$ completely coincide in the defined frequency range. And if the modified flatness parameter $\mathrm{MF}_{P}$ becomes zero, the phase of the modulation index $\arg [\Delta \phi(f)]$ and the target function $\operatorname{TF}_{P}(f)$ completely coincide in that defined frequency range. We tried to find the condition for minimizing the two flatness parameters $\mathrm{MF}_{M}$ and $\mathrm{MF}_{P}$ at the same time by tuning the domain lengths $L_{i}(i=1,2, \ldots, M)$ successively and repeatedly. In this calculation, the initial value for each domain length is set as the same value in the periodic case $L_{i}=L(i=1,2, \ldots, M)$. At the first step of calculation, only the length of the first domain $L_{1}$ is changed, and the corresponding modulation index value is calculated. By repeating the calculation with tuning the length $L_{1}$, the smallest values of $\mathrm{MF}_{M}$ and $\mathrm{MF}_{P}$ which are called the local minimizing values in this calculation step are obtained. After that, only the next domain length $L_{2}$ is tuned and the next local minimal values of $\mathrm{MF}_{M}$ and $\mathrm{MF}_{P}$ are obtained. These local minimal values are smaller than the previous ones, respectively. This calculation step with tuning the domain length is repeated by changing the domain to be tuned from the first domain to the last domain successively. When finishing the calculation step with tuning the last domain length, the domain to be tuned is set to the first domain again and these calculation steps are repeated. In these repeating calculations, the minimal values of the two flatness parameters $\mathrm{MF}_{M}$ and $\mathrm{MF}_{P}$ are obtained when the local minimal values become the same with the values in the previous calculation step, respectively. As a result, the length of each domain is optimal and the difference between the frequency responses of modulation index and the target functions becomes minimum. Therefore, it is possible to control the frequency responses of the magnitude and the phase of modulation index.

\section{Calculation Results}

We tried to calculate the frequency response using our method shown in the previous section. In these calculations, the attenuation coefficient $\alpha$ was set as negligible for simplicity. It is also possible to calculate and design the frequency response of the modulation index taking into account the attenuation coefficient [8].

Figure 3 shows examples of calculated frequency responses of the magnitude and the phase of the modulation index in the traveling-wave electrooptic modulators with nonperiodic polarization reversals for the flat, parabolic magnitude responses and linear phase responses. The number of domains was set as $M=6$ and the frequency range was set from $f_{1}=0.7 f_{m}$ to $f_{2}=1.3 f_{m}$. The target function for the flat magnitude response was defined as $\mathrm{TF}_{M 1}(f)=c_{1}\left(c_{1}\right.$ is constant, and its value is proportional to the applied modulation voltage to the electrodes), and the 


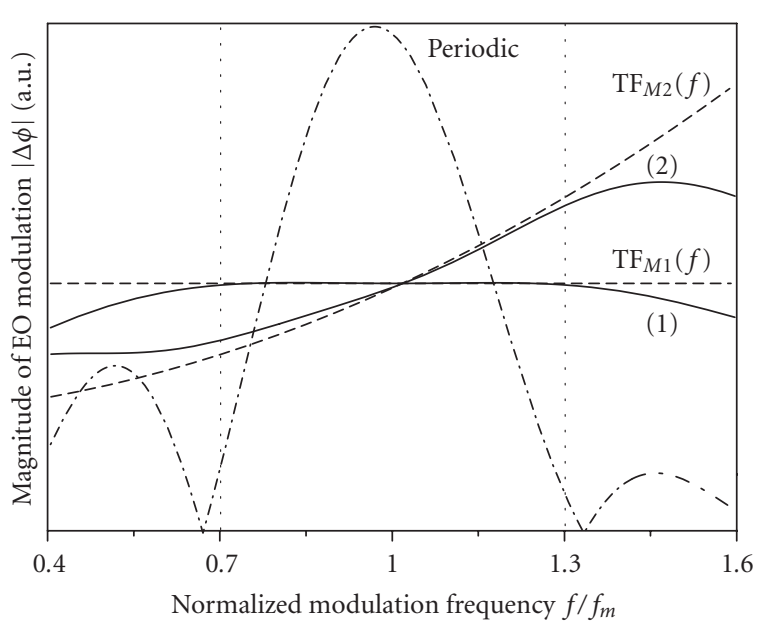

(a)

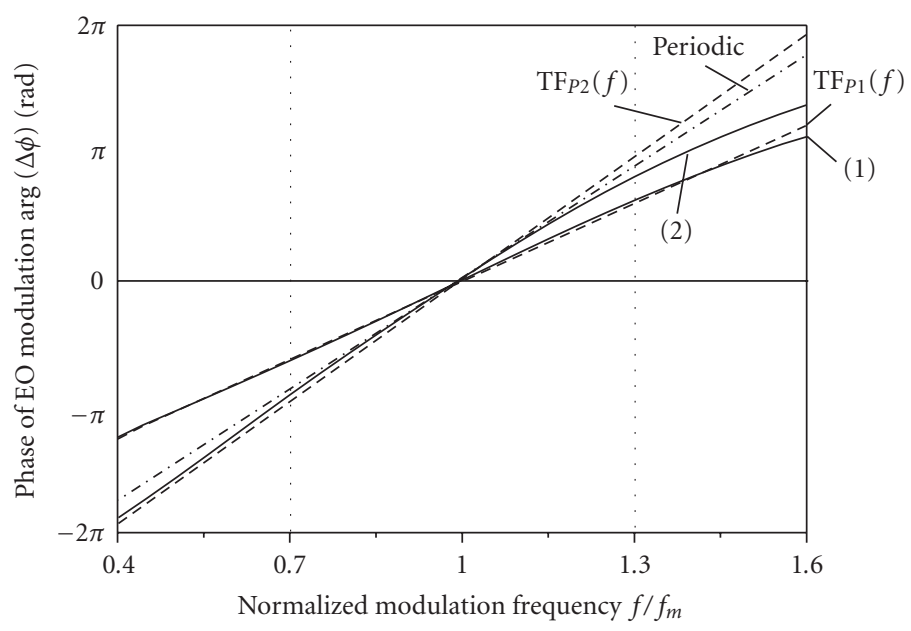

(b)

Figure 3: Frequency dependence of modulation index in traveling-wave electrooptic modulators for flat, parabolic magnitude responses with linear phase responses. (a) Magnitude responses, (b) phase responses.

TABLE 1: (1) Obtained normalized domain lengths for the flat magnitude response and the linear phase response, and (2) the parabolic magnitude response and the linear phase response.

\begin{tabular}{lllllll}
\hline & $L_{1} / L$ & $L_{2} / L$ & $L_{3} / L$ & $L_{4} / L$ & $L_{5} / L$ & $L_{6} / L$ \\
\hline$(1)$ & 0.03 & 0.22 & 0.41 & 0.40 & 1.07 & 0.77 \\
(2) & 0.22 & 0.41 & 0.58 & 0.58 & 0.77 & 0.96 \\
\hline
\end{tabular}

target function for the linear phase response was defined as $\mathrm{TF}_{P 1}(f)=a_{1}\left[\left(f / f_{m}\right)-1\right]\left(a_{1}\right.$ is constant, and it is set as 6.5 rad in calculation). With these conditions, the calculation for minimizing the flatness parameters was carried out. The obtained frequency responses of the magnitude and the phase are shown by the curves (1) in Figures 3(a) and 3(b), respectively.

The target function for the parabolic magnitude response was also defined as $\operatorname{TF}_{M 2}(f)=c_{2}\left[\left(f / f_{m}\right)^{2}-1\right]+c_{1}$ (with $c_{2} / c_{1}$ is set as 0.6$)$ and the target function for the linear phase response was defined as $\operatorname{TF}_{P 2}(f)=a_{2}\left[\left(f / f_{m}\right)-1\right]$ (with $a_{2}$ is set as $10 \mathrm{rad}$ ). The calculation was also carried out. The obtained frequency responses of the magnitude and the phase are also shown as the curves (2) in Figures 3 (a) and 3(b), respectively. For comparison, the frequency responses of the magnitude and the phase in travelingwave electrooptic modulator with the periodic polarization reversal are also shown in the figures.

The obtained frequency responses almost coincide with the target functions in the designed frequency ranges. Table 1 shows the values of the obtained domain lengths normalized by the length $L$. The length $L$ is the domain length in the periodic polarization reversal for QVM at the peak modulation frequency $f_{m}$. For example, if we set the peak modulation frequency as $f_{m}=15 \mathrm{GHz}$ and the lightwave wavelength as $\lambda=1.55 \mu \mathrm{m}$, then the corresponding length of $L$ becomes $L=4.33 \mathrm{~mm}$ for a $\mathrm{LiTaO}_{3}$ guided-wave modulator (microwave phase velocity $v_{m}=6.47 \times 10^{7} \mathrm{~m} / \mathrm{s}$ and lightwave group velocity $v_{g}=1.29 \times 10^{8} \mathrm{~m} / \mathrm{s}$ ).

We confirmed the validity of the proposed method. It is possible to obtain the traveling-wave electrooptic modulators with the required frequency responses of both the magnitude and the phase of the modulation index by using our new approach.

\section{Applications}

Recently, there are many reports related to the advanced modulation formats such as duobinary, SSB, QPSK modulation to increase the capacity of the data transmission for the new generation optical fiber communication systems. Using the control technique of the frequency responses in the electrooptic modulators with the nonperiodic polarization reversal, it is possible to design the new electrooptic modulators for the advanced modulation formats.

\subsection{Electrooptic Intensity Modulator for Duobinary Modulation}

The optical duobinary modulation format offers a larger capacity of data transmission and a large tolerance for fiber chromatic dispersion in optical fiber communication system $[3,10]$. Several experimental studies on the generation of the optical duobinary modulation signals have been reported. In these reports, the electrical/optical filters are required to limit the spectrum bandwidth of the generated signals. By using the nonperiodically polarization-reversed structure, it is possible to obtain the electrooptic modulators with specifically tuned filter-like modulation frequency responses with any passband (low-pass, high-pass, band-pass, Gaussian like, etc.). It can be used for the generation of the duobinary modulation signals without the external electric/optic filters. 


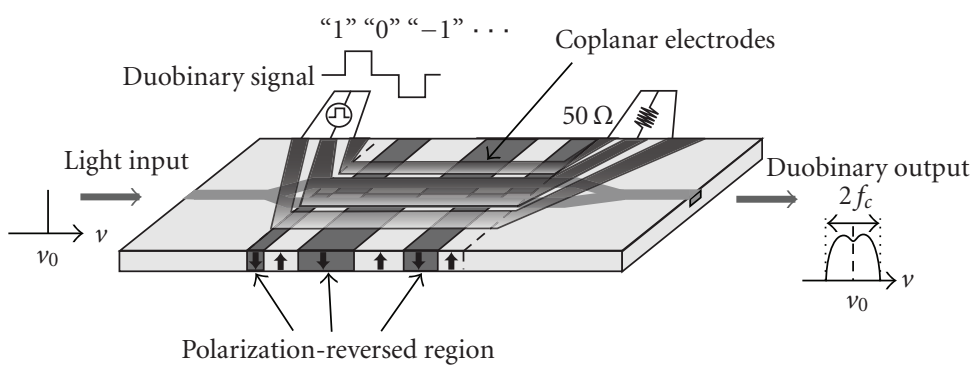

(a)

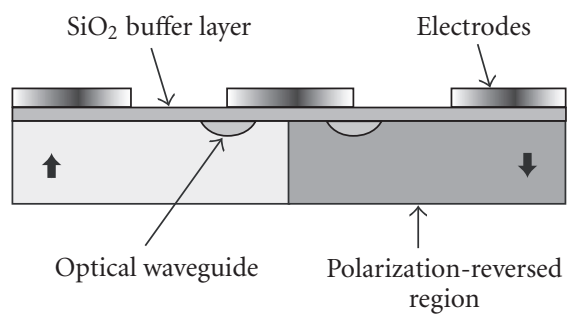

(b)

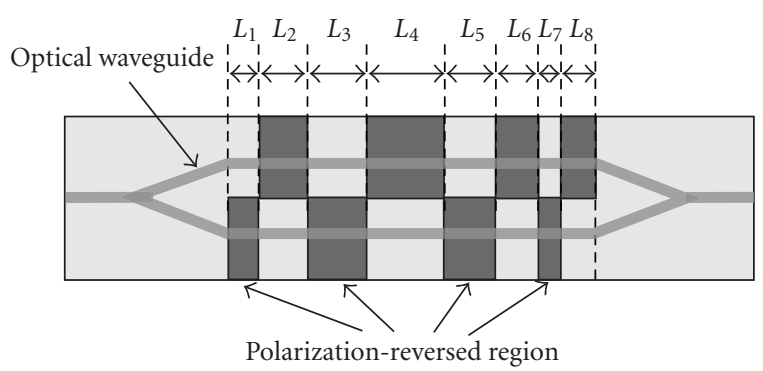

(c)

Figure 4: Basic structure of duobinary modulator with nonperiodic polarization reversal. (a) Whole view, (b) cross-section view, (c) polarization-reversed patterns.

Figure 4 shows the structure of the electrooptic intensity modulator for duobinary modulation using nonperiodic polarization reversal. It consists of the Mach-Zehnder waveguide, coplanar traveling-wave electrodes, and nonperiodically polarization-reversed structures. The lengths of each domain are the same in the two arms of the Mach-Zehnder waveguide, however, the polarity is opposite in the two arms. Therefore, by applying the electrical fields with the same direction, the push-pull intensity modulation with the same modulation index magnitude for the duobinary operation is obtained with a single modulation signal.

Using the proposed method for the frequency response control, we tried to obtain a Gaussian filter-like frequency response in the electrooptic modulation with a cutoff frequency $f_{c}$ for generating the optical duobinary modulation signals. The target function for the Gaussian filter-like magnitude response was defined as $\operatorname{TF}_{\mathrm{MG}}(f)=$ $\Delta \phi_{0} \exp \left[-g\left(f / f_{m}\right)^{2}\right]$ (with $g$ is set as 2) and the target function for the linear phase response was defined as $\operatorname{TF}_{\mathrm{PG}}(f)=a\left(f / f_{m}\right)$ (with $a$ is set as $5.5 \mathrm{rad}$ ). The number of domains was set as $M=8$ and the frequency range was set as the baseband from $f_{1}=0$ to $f_{2}=f_{m}$. The obtained results are shown in Figure 5. The targets functions are shown as

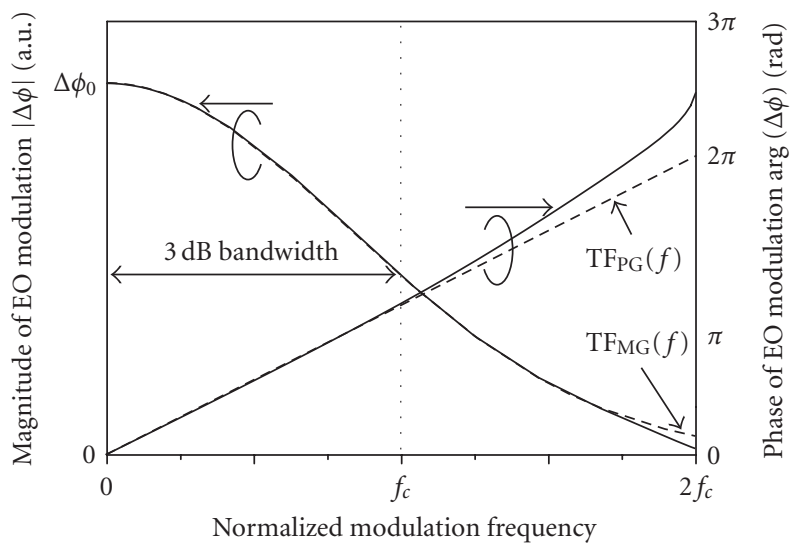

FIGURE 5: Frequency dependence of modulation index in travelingwave electrooptic modulator for the Gaussian-like filter magnitude response and the linear phase response.

the dashed lines. The obtained frequency responses of the magnitude and the phase of electrooptic modulation are shown as the solid lines. Table 2 shows the obtained domain 


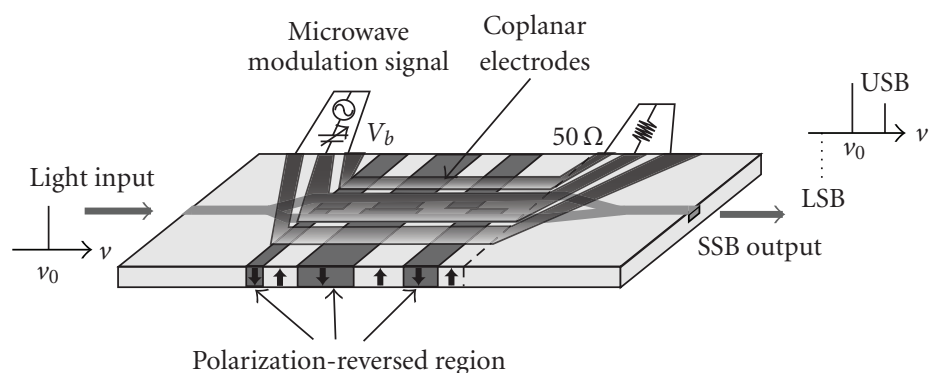

(a)

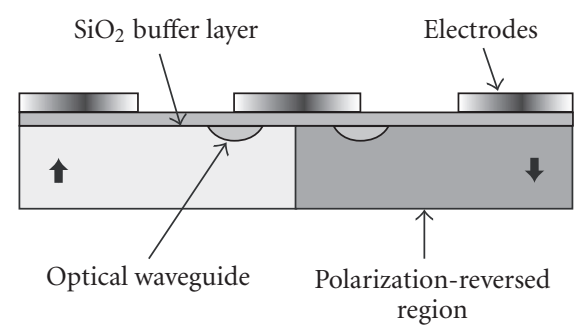

(b)

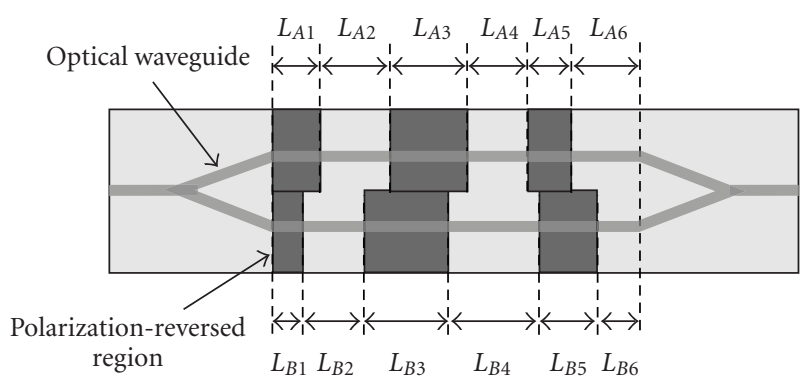

(c)

FIGURE 6: Basic structure of SSB modulator with nonperiodic polarization reversal. (a) Whole view, (b) cross-section view, (c) polarizationreversed patterns.

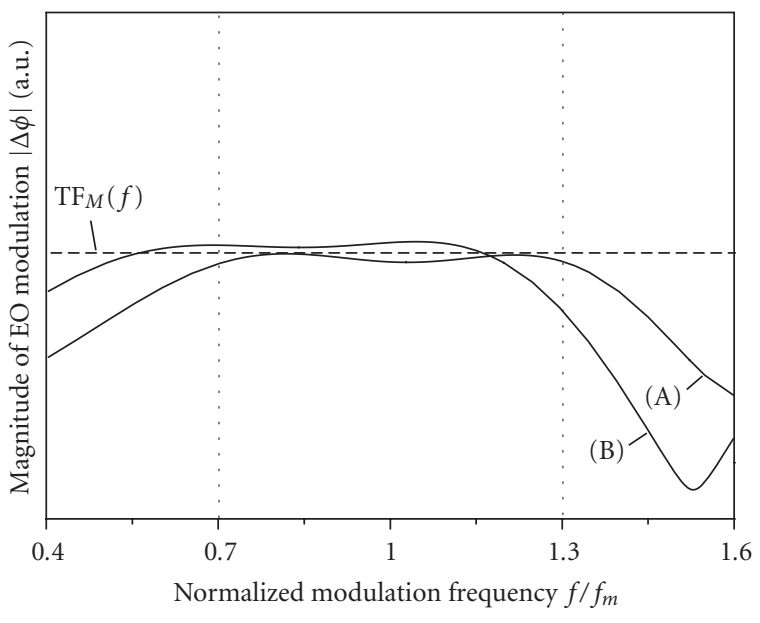

(a)

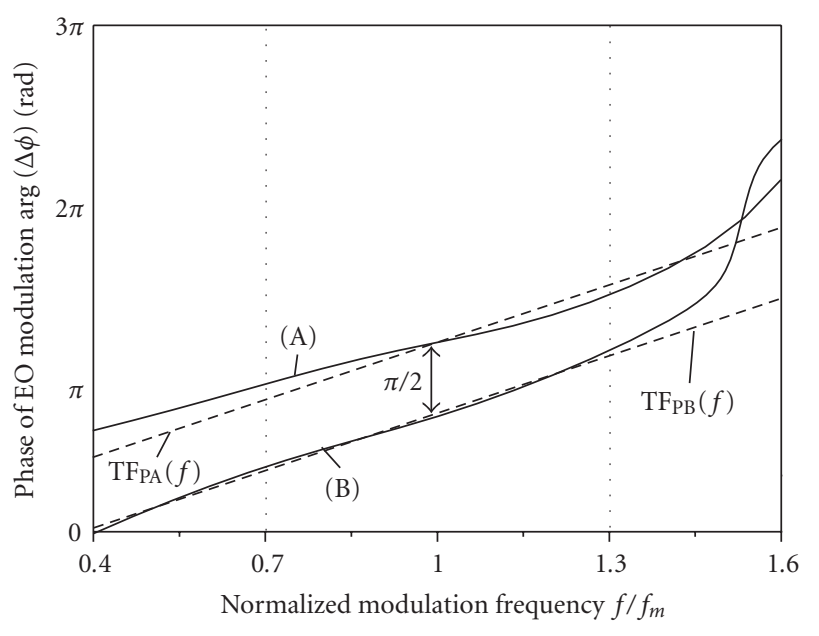

(b)

FIGURE 7: Frequency dependence of modulation index in designed SSB modulator for flat magnitude responses and linear phase responses with $\pi / 2$ difference. (a) Magnitude responses, (b) phase responses. 
TABLE 2: Obtained normalized domain lengths for the Gaussianlike magnitude response and the linear phase response.

\begin{tabular}{lccccccc}
\hline$L_{1} / L$ & $L_{2} / L$ & $L_{3} / L$ & $L_{4} / L$ & $L_{5} / L$ & $L_{6} / L$ & $L_{7} / L$ & $L_{8} / L$ \\
0.102 & 0.359 & 0.281 & 1.999 & 0.100 & 0.101 & 0.159 & 0.111 \\
\hline
\end{tabular}

lengths normalized by the periodic case length $L$. By using the Gaussian filter-like magnitude and the linear phase responses in electrooptic modulation, it is possible to generate the duobinary modulation signals by simple digital electrical driving signal without any filter.

We also applied the calculated polarization reversal pattern into the two arms of the Mach-Zehnder waveguide in the proposed duobinary modulator and calculated its performance. For the design of a $10 \mathrm{Gbps}$ duobinary modulator, the cutoff frequency $f_{c}$ was set as $5 \mathrm{GHz}$ and the lightwave wavelength as $\lambda=1.55 \mu \mathrm{m}$. Then, the total electrode length $L_{t}$ became $L_{t}=24 \mathrm{~mm}$ for a $\mathrm{LiTaO}_{3}$-based device.

We also checked the effect caused by the attenuation of the modulation microwave in the electrodes. We assumed a typical attenuation coefficient of the modulation microwave $\left(\alpha=0.25 \mathrm{~dB} / \mathrm{cm} / \mathrm{GHz}^{0.5}\right)$ in coplanar electrodes. As a result, the frequency response of the 10 Gbps duobinary modulator with the microwave attenuation became almost the same with the calculated one with the negligible attenuation coefficient in the frequency range from 0 to $10 \mathrm{GHz}$. Therefore, an $\sim 10$ Gbps duobinary modulator is expected using our new approach.

\subsection{Broadband Single-Sideband Modulator}

By using optical single-sideband modulation formats, it is possible to reduce the optical power in the signal transmission. This is attractive in long-haul optical fiber communication systems with higher density wavelength multiplexing [4]. We proposed and demonstrated the SSB modulators using the periodic polarization reversal [6]. By using our new approach, the SSB modulator with the flat and wider operational frequency range can be designed.

Figure 6 shows the structure of this electrooptic modulator using nonperiodic polarization reversal. It consists of the Mach-Zehnder waveguide, coplanar traveling-wave electrodes, and two nonperiodically polarization-reversed patterns set in the two arms of the Mach-Zehnder waveguide. In order to have the SSB modulation characteristics, it is required that the magnitudes of the modulation index are the same in the two arms of the Mach-Zehnder waveguide and the phases of the modulation index in the two arms are different as $\pi / 2$ in the designed frequency range. For realizing these two modulation characteristics, we applied the control method of the frequency responses.

Figure 7 shows the calculated frequency responses for the SSB modulator with nonperiodic polarization reversal for the same target function $\operatorname{TF}_{\mathrm{MA}}(f)=\mathrm{TF}_{\mathrm{MB}}(f)=\mathrm{TF}_{M}(f)=$ $c$ (constant) and linear target functions $\operatorname{TF}_{\mathrm{PA}}(f)=a\left(f / f_{m}\right)+$ $b$ and $\operatorname{TF}_{\mathrm{PB}}(f)=a\left(f / f_{m}\right)+b-\pi / 2$ (with $a=4 \mathrm{rad}$, $b$ is a common phase offset value). In this example, the number of domains was set as $M=6$ and the frequency

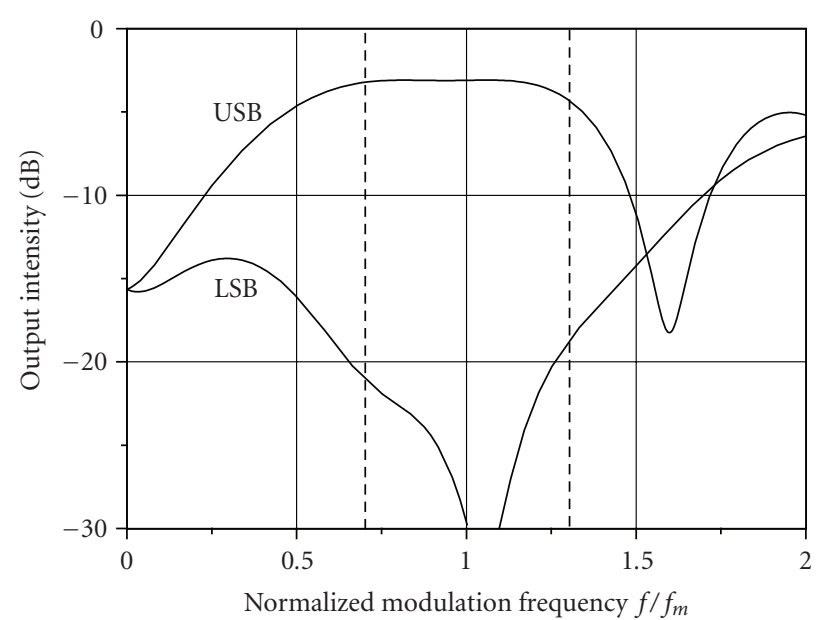

FIGURE 8: Frequency responses of USB and LSB components of designed SSB modulator.

TABLE 3: Obtained normalized domain lengths for the designed SSB modulator.

\begin{tabular}{lcccccc}
\hline & $L_{1} / L$ & $L_{2} / L$ & $L_{3} / L$ & $L_{4} / L$ & $L_{5} / L$ & $L_{6} / L$ \\
\hline (A) & 0.65 & 1.31 & 0.58 & 0.51 & 0.48 & 0.28 \\
(B) & 0.19 & 0.96 & 1.42 & 0.45 & 0.51 & 0.24 \\
\hline
\end{tabular}

range was set as $f_{1}=0.7 f_{m}$ and $f_{2}=1.3 f_{m}$. The calculated frequency responses of the magnitude and the phase of modulation index are shown by curves (A) and (B) in Figure 7. The obtained frequency responses of the modulation index magnitudes are flat and almost the same. The obtained frequency responses of the modulation index phase are linear and have a phase difference of about $\pi / 2$ in the designed frequency range. Table 3 shows the obtained domain lengths normalized by the periodic case length $L$.

We applied the calculated polarization reversal patterns (A) and (B) into the two arms of the Mach-Zehnder waveguide in the SSB modulator and calculated the performance of the SSB modulation. The obtained frequency dependence of the upper sideband (USB) and the lower sideband (LSB) is shown in Figure 8, where we assumed the optical delay of $\lambda / 4$ in the two arms of the Mach-Zehnder waveguide by supplying an appropriate DC bias voltage to the electrodes. It is shown that the USB is generated around the designed frequency range, while the LSB is almost suppressed. The sideband suppression is expected over $20 \mathrm{~dB}$ around the designed frequency range. The characteristics of the USB and the LSB can be switched by changing the DC bias voltage. Therefore, the broadband SSB modulation characteristics are expected with our new approach.

\section{Conclusion}

We proposed the novel design of the traveling-wave electrooptic modulators using nonperiodically polarizationreversed structures to control frequency responses of both the magnitude and the phase of the modulation index. It is 
possible to design advanced electrooptic modulators such as duobinary modulators or broadband SSB modulators. This approach is also applicable to design other devices such as broadband optical frequency-shift-keying (FSK) modulator, and broadband optical frequency shifter (OFS).

\section{Acknowledgments}

This work was supported in part by Grants-in-Aid for Scientific Research from the Ministry of Education, Culture, Sports, Science, and Technology of Japan. This research was also partially support by a grant from the Global COE Program, "Center for Electronic Devices Innovation," from the Ministry of Education, Culture, Sports, Science, and Technology of Japan.

\section{References}

[1] E. L. Wooten, K. M. Kissa, A. Yi-Yan, et al., "A review of lithium niobate modulators for fiber-optic communications systems," IEEE Journal of Selected Topics in Quantum Electronics, vol. 6, no. 1, pp. 69-82, 2000.

[2] T. Kawanishi, T. Sakamoto, and M. Izutsu, "High-speed control of lightwave amplitude, phase, and frequency by use of electrooptic effect," IEEE Journal of Selected Topics in Quantum Electronics, vol. 13, no. 1, pp. 79-91, 2007.

[3] K. Yonenaga and S. Kuwano, "Dispersion-tolerant optical transmission system using duobinary transmitter and binary receiver," Journal of Lightwave Technology, vol. 15, no. 8, pp. 1530-1537, 1997.

[4] S. Shimotsu, S. Oikawa, T. Saitou, et al., "Single side-band modulation performance of a $\mathrm{LiNbO}_{3}$ integrated modulator consisting of four-phase modulator waveguides," IEEE Photonics Technology Letters, vol. 13, no. 4, pp. 364-366, 2001.

[5] H. Murata, K. Kinoshita, G. Miyaji, A. Morimoto, and T. Kobayashi, "Quasi-velocity-matched $\mathrm{LiTaO}_{3}$ guided-wave optical phase modulator for integrated ultrashort optical pulse generators," Electronics Letters, vol. 36, no. 17, pp. 1459-1460, 2000 .

[6] H. Murata, K. Kaneda, and Y. Okamura, " $38 \mathrm{GHz}$ optical single-sideband modulation by using guided-wave electrooptic modulators with periodic polarization-reversal," in Proceedings of Conference on Lasers and Electro-Optics (CLEO '04), vol. 2, p. 3, San Francisco, Calif, USA, May 2004.

[7] H. Murata, T. Iwamoto, and Y. Okamura, "Novel electrooptic optical frequency shifters by use of 3-branch waveguide interferometer and polarization-reversal structures," in Proceedings of the 9th Optoelectronics and Communications Conference (OECC '04), pp. 564-565, New York, NY, USA, July 2004.

[8] H. V. Pham, H. Murata, and Y. Okamura, "Travelling-wave electrooptic modulators with arbitrary frequency response utilising non-periodic polarisation reversal," Electronics Letters, vol. 43, no. 24, pp. 1379-1381, 2007.

[9] H. Murata, A. Morimoto, T. Kobayashi, and S. Yamamoto, "Optical pulse generation by electrooptic-modulation method and its application to integrated ultrashort pulse generators," IEEE Journal of Selected Topics in Quantum Electronics, vol. 6, no. 6, pp. 1325-1331, 2000.

[10] D. M. Gill, A. H. Gnauck, X. Liu, et al., “42.7-Gb/s costeffective duobinary optical transmitter using a commercial 10$\mathrm{Gb} / \mathrm{s}$ mach-zehnder modulator with optical filtering," IEEE Photonics Technology Letters, vol. 17, no. 4, pp. 917-919, 2005. 

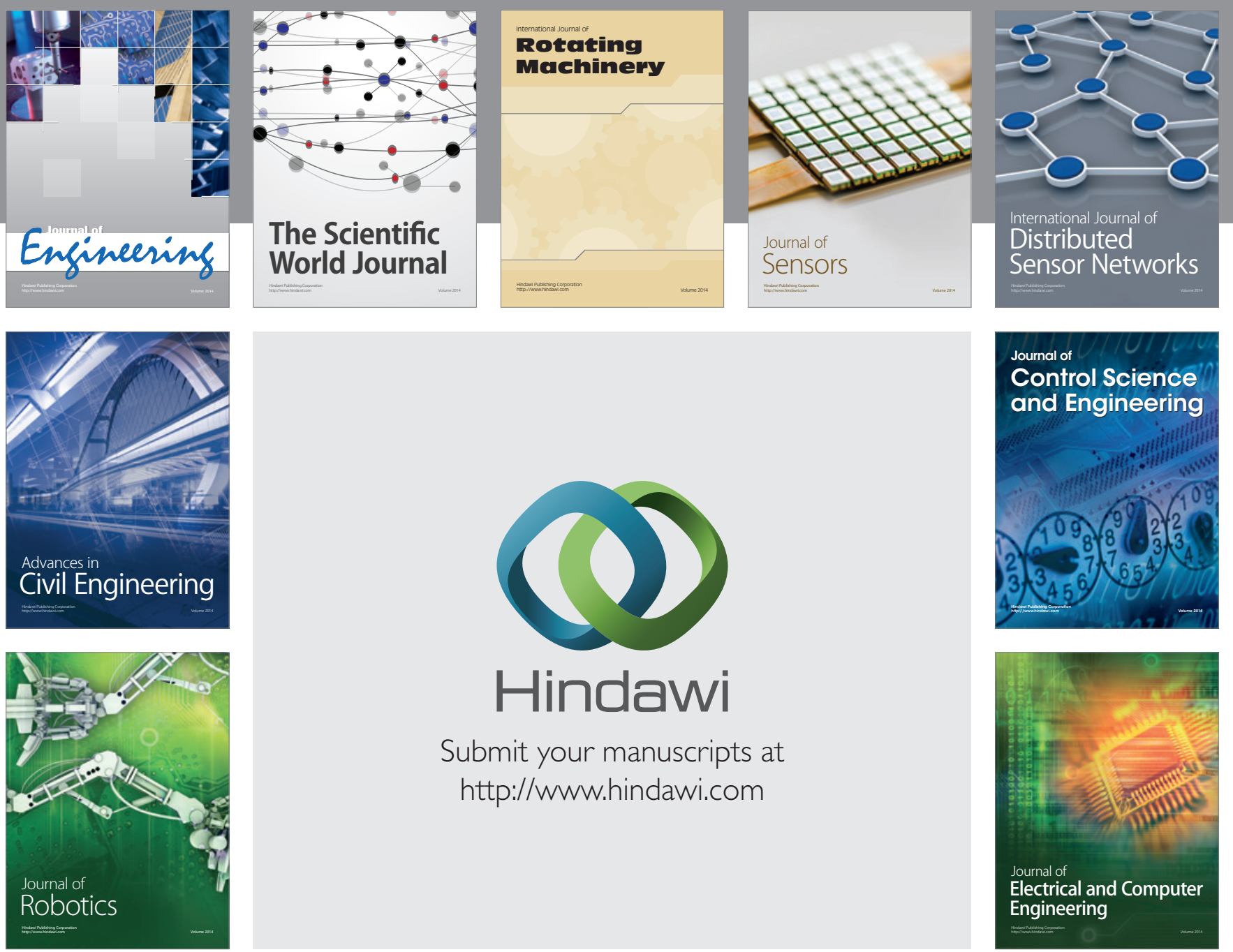

Submit your manuscripts at

http://www.hindawi.com
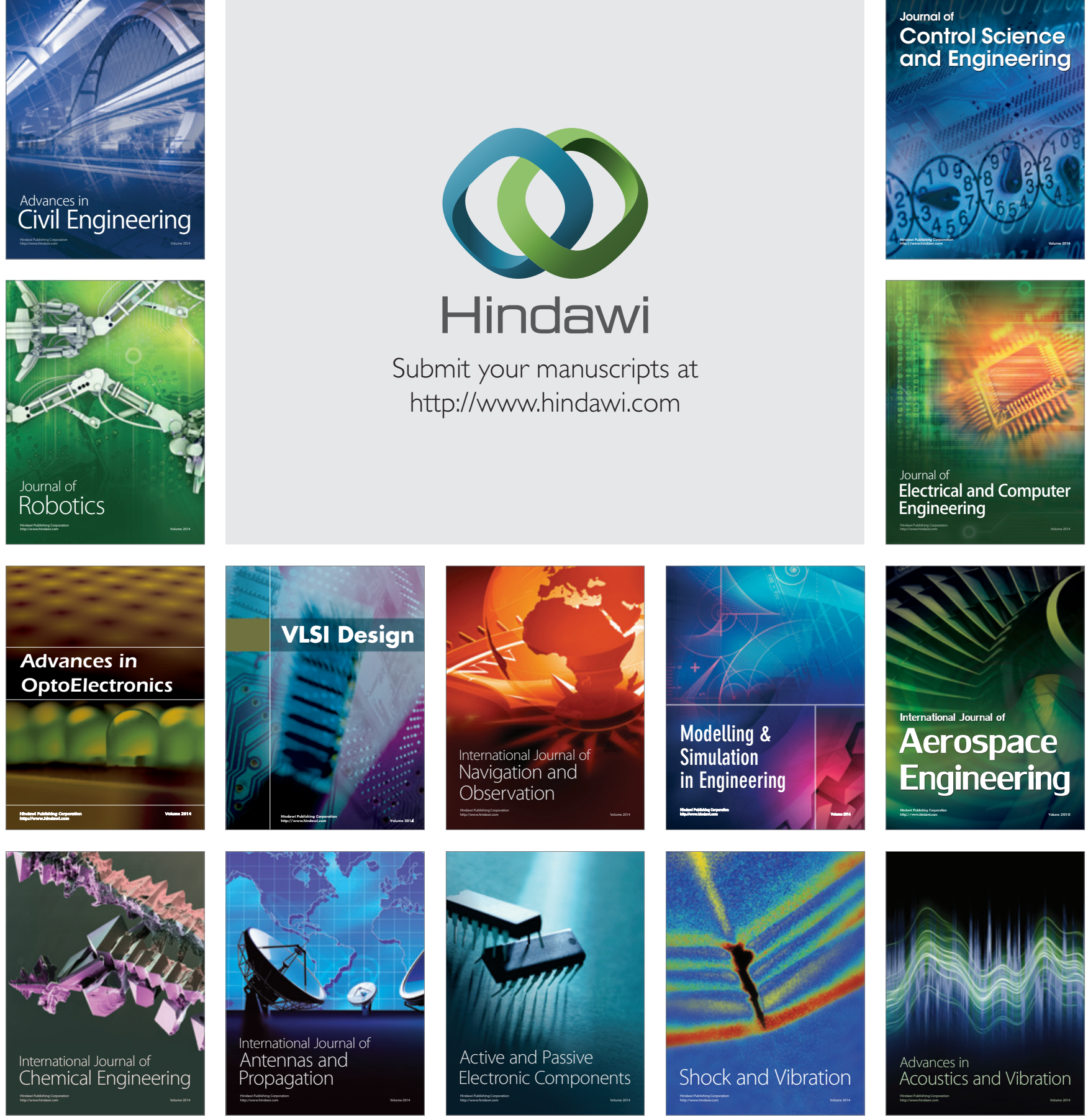